\title{
Continuing doubt on gravitation
}

\section{The latest measurement of the gravitational constant does not improve the precision with which this important quantity is known. Is there a more radical approach?}

EMBARRASSMENT at the difficulty of obtaining an accurate value of the gravitational constant $(G)$ has long since ceased to be a joke. So much is clear from A.H. Cook's latest attempt at a measurement of $G$, now published (Y.T. Chen, A.H. Cook and A.J.F. Metherell, Proc. $R$. Soc. A 394, 47-68; 1984). Part of the joke is that Cook and his colleagues at the Cavendish Laboratory, Cambridge, set out to measure not $G$, the constant in the inverse-square law of gravitational force, but departures from Newton's law of gravitation. Their conclusion is that the inverse-square law holds even more precisely than had been thought, but their implied value of $G$ is hardly more accurate than that of their predecessors in the measurement of this most elusive constant.

The position is quite tantalizing. Nowadays the strength of gravitational forces in the neighbourhood of substantial masses such as the Sun or the Earth can be estimated with increasing accuracy. Measurement of Earth-satellite orbits, for example, can now yield the value of $G M$ (where $M$ is the mass of the Earth) to within one part in $10^{7}$, essentially the precision of its distance measurements. But the value of $G$ remains obdurately uncertain to the tune of one part in $10^{5}$. The consequences include enormous absolute uncertainties in, say, the mass of the Earth (see Nature 302,$11 ; 1983$ ).

None of this diminishes the cleverness of what Cook's team has been up to. In the trade, the measurement will be known as a torsion balance experiment, in the tradition begun by Cavendish at the end of the eighteenth century which has the advantage that timing self-oscillations obviates the need to know the mechanical properties of the dispersion. In this case, the beam of the torsion balance is a $60-\mathrm{cm}$ aluminium tube suspended by a tungsten fibre nearly a metre long and $75 \mu \mathrm{m}$ in diameter: much of the effort underlying the experiment has gone into the annealing of this filament, in a process devised so as to eliminate dislocations from the crystal lattice and which occupies roughly one month for each fibre. The torsion balance itself has a spherical mass suspended from one end and is entirely enclosed in a vacuum vessel. Electric and magnetic fields are excluded by shielding.

Making measurements with this system is far from being child's play. The torsion balance is fitted with a 3-m optical lever consisting of a beam from an He-Ne laser whose angular deflection, detected by a photocell, can be measured to a millionth of a degree. To ensure the stability (and integrity) of the balance, there is a pair of electrostatic plates at the blind end of the balance beam and an electronic servosystem so as to provide appropriate negative feedback.

Breaking with tradition, the Cambridge team has used as masses for deflecting the test-mass hanging from the balance beam neither spheres (Cavendish) nor annular rings (Long, 1977) but cylinders, arranged with their axes parallel to the balance beam. The calculation is that such an arrangement should be relatively insensitive to displacements in the accurate positioning of the cylindrical masses along their longitudinal axes.

Cook and his colleagues have been to some extent constrained in the design of their equipment by their underlying objective, to measure departures from the inverse-square law. For the past decade, there has been a train of speculation that if gravitational forces are ever to be explained as are the three other kinds of natural forces between material particles, by some mechanism entailing the mutual exchange of field particles of some kind, there will be a small exponential correction to the inverse-square law at sufficiently small distances. D.R. Long startled some of his colleagues nearly a decade ago (Nature 260, $417 ; 1976$ ) by claiming that there are indeed departures from the inverse-square law corresponding to a characteristic distance of $2 \mathrm{~m}$. Detecting such variations from ideality evidently requires substantial separations between the gravitating masses. The benefit is that Long's characteristic distance has been pushed out to between 3 and $4 \mathrm{~m}$. The snag is that the uncertainty of $G$ implied by the measurements is greater than that of the most accurate measurement so far reported, that of G.G. Luther and W.R. Towler (Phys. Rev. Lett. 48, 121; 1982) - in their torsion balance experiment.

Two general questions arise, of which the most immediate may be the difficulty of making substantially more accurate measurements. There are two longstanding obstacles to further refinement the fact that gravitational forces are indeed much weaker than other kinds of forces, so that it is not possible to think of balancing gravitational and, say, electromagnetic forces in some clever electronic experiment, and the awkward circumstance that the gravitational force between two masses depends on the product of the two. In measurements such as that of Cook's group, it is beneficial in some ways that test-mass hung from the balance beam need never be directly measured, but the consequence is that everything turns on the ratio of the masses of two external objects, which boils down to a problem in macroscopic metrology - and the assumption that the deflecting objects are homogeneous and identical in composition.

The time therefore seems ripe for a radically new approach to the design of measurements of $G$. The temptation to look for oscillating mechanical systems where masses might be made measurable by some resonance phenomenon should be resisted; the mass of the supports needed to keep an oscillating system stable would probably dominate the measurement. Levitating superconducting objects magnetically would have the disadvantage that they might be made into little dynamos. Bending beams of neutrons might do the trick if it were possible to measure velocity of momentum with sufficient accuracy.

Meanwhile, uncertainty about the correct value of $G$ will persist. The internationally accepted CODATA value is $6.6720 \pm$ $0.0041 \times 10^{-11} \mathrm{~m}^{3} \mathrm{~kg}^{-1} \mathrm{~s}^{-2}$. George $\mathrm{T}$. Gillies, at the Bureau International des Poids et Mesures, who has shouldered the task of keeping track of $G$ and related measurements, points out that the three most recent values claimed to have superior accuracy (one part in $10^{5}$ ) differ among themselves by more than any of the standard deviations claimed. It will not be easy to wring an agreed value from these measurements, or even to look for a precision much exceeding one part in 5,000.

According to Gillies' latest review of the position, completed in 1983, there is much less doubt about other kinds of measurments made of quantities related to $G$. From time to time, people have been concerned with questions as odd as the permeability of other materials for the gravitational force, the variation of gravitational force with condition of interacting masses and even the temperature variation. Most of the results are, of course, null results. There are signs of life only in the question whether $G$ varies systematically with time, as first suggested by Dirac. The search for time variation $G$ has recruited a small army of devotees, but that is another story. John Maddox 